\title{
TWO-DIMENSIONAL FLUID-HAMMER ANALYSIS BY THE METHOD OF NEARCHARACTERISTICS \\ by
}

Yong W. Shin and Christian A. Kot

BASE TECHNOLOGY
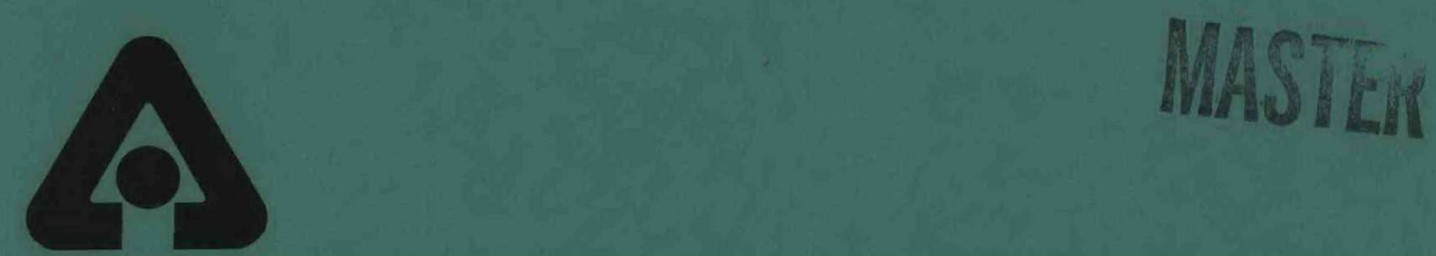

U OF C-AUA-USEROA

ARGONNE NATIONAL LABORATORY, ARGONNE, ILLINOIS

Prepared for the U.S. ENERGY RESEARCH

AND DEVELOPMENT ADMINISTRATION

Division of Reactor Research and Development

under Contract W-31-109-Eng-38 


\section{DISCLAIMER}

This report was prepared as an account of work sponsored by an agency of the United States Government. Neither the United States Government nor any agency Thereof, nor any of their employees, makes any warranty, express or implied, or assumes any legal liability or responsibility for the accuracy, completeness, or usefulness of any information, apparatus, product, or process disclosed, or represents that its use would not infringe privately owned rights. Reference herein to any specific commercial product, process, or service by trade name, trademark, manufacturer, or otherwise does not necessarily constitute or imply its endorsement, recommendation, or favoring by the United States Government or any agency thereof. The views and opinions of authors expressed herein do not necessarily state or reflect those of the United States Government or any agency thereof. 


\section{DISCLAIMER}

Portions of this document may be illegible in electronic image products. Images are produced from the best available original document. 
The facilities of Argonne National Laboratory are owned by the United States Government. Under the terms of a contract (W-31-109-Eng-38) between the U. S. Energy Research and Development Administration, Argonne Universities Association and The University of Chicago, the University employs the staff and operates the Laboratory in accordance with policies and programs formulated, approved and reviewed by the Association

\section{MEMBERS OF ARGONNE UNIVERSITIES ASSOCIATION}

The University of Arizona Carnegie-Mellon University Case Western Reserve University The University of Chicago University of Cincinnati Illinois Institute of Technology University of Illinois Indiana University Iowa State University The University of Iowa
Kansas State University The University of Kansas Loyola University Marquette University Michigan State University The University of Michigan University of Minnesota University of Missouri Northwestern University University of Notre Dame
The Ohio State University Ohio University The Pennsylvania State University Purdue University Saint Louis University Southern Illinois University The University of Texas at Austin Washington University Wayne State University The University of Wisconsin

This report was prepared as an account of work sponsored by the United States Government. Neither the United States nor the United States Energy Research and Development Administration, nor any of their employees, nor any of their contractors, subcontractors, or their employees, makes any warranty, express or implied, or assumes any legal liability or responsibility for the accuracy, completeness or usefulness of any information, apparatus, product or process disclosed, or represents that its use would not infringe privately-owned rights. Mention of commercial products, their manufacturers, or their suppliers in this publication does not imply or connote approval or disapproval of the product by Argonne National Laboratory or the U. S. Energy Research and Development Administration.

Printed in the United States of America

Available from

National Technical Information Service

U. S. Department of Commerce

5285 Port Royal Road

Springfield, Virginia 22161

Price: Printed Copy $\$ 4.00 ;$ Microfiche $\$ 2.25$ 
Distribution Category:

LMFBR Structural Materials and Design Engineering (UC-79h)

ANL-75-21

\section{ARGONNE NATIONAL LABORATORY}

9700 South Cass Avenue

Argonne, Illinois 60439

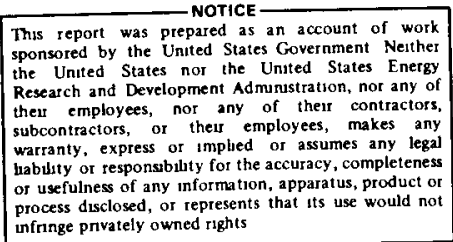

TWO-DIMENSIONAL FLUID-HAMMER ANALYSIS BY THE METHOD OF NEARC HARACTERISTICS

by

Yong W. Shin and Christian A. Kot

Components Technology Division

May 1975 
$\bullet$ 


\section{TABLE OF CONTENTS}

Page

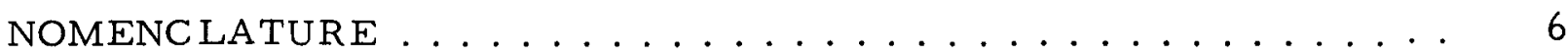

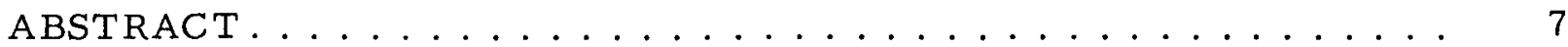

I. INTRODUCTION. ..................... 7

II. BASIC DIFFERENTIAL EQUATIONS .............. 8

III. NEARCHARACTERISTIC FORMULATION. . . . . . . . . 10

IV. BOUNDARY AND AXIAL-POINT CONDITION . . . . . . . . 12

V. NUMERICAL PROCEDURE ................. 12

VI. NUMERICAL STABILITY. . . . . . . . . . . . 13

VII. NUMERICAL CALCULATIONS . . . . . . . . . . . . . . 15

VIII. DISCUSSIONS AND CONCLUSION . . . . . . . . . . . . 20

APPENDIXES

A. Compatibility Conditions along Nearcharacteristics. . . . . . 22

B. Fourier Stability Analysis of vonNeumann . . . . . . . . . 24

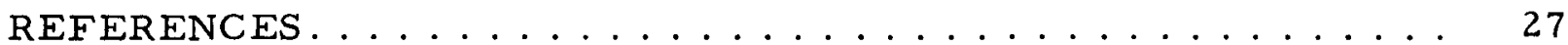




\section{LIST OF FIGURES}

No.

Title

Page

1. Projection on $\mathbf{r}-\mathbf{z}$ Plane of Characteristic Cone and Nearcharacteristics .................... 12

2. Finite-difference Network. ................. 14

3. Diffraction of Plane Acoustic Wave from $90^{\circ}$ Sharp Wedge..... 15

4. Pressure-Time History of Points Fixed on Wedge Faces . . . . 16

5. Sample Problem ....................... 16

6. Nondimensional Pressure at Point G Obtained by Three Numerical Schemes . . . . . . . . . . . . . . . . 17

7. Nondimensional Pressure at Point G Comparing Nearcharacteristic with Bicharacteristic Solution. ............. 17

8. Nondimensional Pressure at Point $\mathrm{K}$ Comparing Nearcharacter istic with Bicharacteristic Solution. .............. 18

9. Nondimensional Pressure at Point H Comparing Nearcharacteristic with Bicharacteristic Solution. . . . . . . . . . . 18

10. Nondimensional Pressure at Point G Comparing Nonlinear with Linear Solution . . . . . . . . . . . . . . . . . . .

11. Nearcharacteristic Solution at Points $G$ and $K$ in Comparison with One-dimensional Acoustic and Numerical Solutions.......

12. Nearcharacteristic Solution at Point $\mathrm{H}$ in Comparison with Onedimensional Acoustic and Numerical Solutions . . . . . . . . . . 
0

0

OMENCLATURE 
NOMENC LATURE

b Aspect ratio defined in Eq. $9 \mathrm{~b}$

Fluid-hammer speed

L Characteristic length in $\mathrm{z}$ coordinate

$P \quad$ Vertex of characteristic cone

p Pressure

$\Delta \mathrm{p} \quad$ Pressure pulse used as reference pressure

Radial coordinate

$\Delta \mathrm{r} \quad$ Grid size in $\mathrm{r}$ coordinate

$\mathrm{R} \quad$ Characteristic length in $\mathrm{r}$ coordinate

s Entropy

t Time

$\Delta t \quad$ Time step

u Velocity component in $r$ coordinate

$\mathrm{U}$ Characteristic velocity for both $\mathrm{r}$ and $\mathrm{z}$ coordinates

$\mathrm{w} \quad$ Velocity component in $\mathrm{z}$ coordinate

Axial coordinate

$\Delta z \quad$ Grid size in $z$ coordinate

e Nonlinearity parameter defined in Eq. $9 a$

$\nu \quad$ Cartesian if $\nu=0$ and cylindrical geometry if $\nu=1$

p Density 


\title{
TWO-DIMENSIONAL FLUID-HAMMER ANALYSIS \\ BY THE METHOD OF NEARCHARACTERISTICS
}

by

Yong W. Shin and Christian A. Kot

\begin{abstract}
A numerical technique based on the method of nearcharacteristics is considered for solving propagation of fluidhammer waves in a two-dimensional geometry. The solution is constructed by relating flow conditions by compatibility equations along lines called nearcharacteristics. Three choices are considered in the numerical scheme that are accurate within an error of the order of magnitude of the time step.

Since the nearcharacteristics lie in the coordinate planes, the technique provides an efficient method requiring only simple interpolations in the initial plane. On the other hand, the nearcharacteristics fall outside the characteristic cone. Thus the solution procedure directly refers to conditions outside the true domain of dependence. The effect of this is studied through numerical calculation of a simple example problem and comparison with results obtained by a bicharacteristic method. Comparison is also made with existing analytical solutions and experiments. Furthermore, the three solution schemes considered are examined for numerical stability by the vonNeumann test. Two of the schemes were found to be unstable; the third yielded a stability criterion equivalent to that of the bicharacteristic formulation. The stabilityanalysis results were confirmed by numerical experimentation.
\end{abstract}

\section{INTRODUCTION}

The method of characteristics is an efficient and informative technique for numerical integration of quasilinear hyperbolic systems of differential equations. The equations are cast in a form that relates the conditions along lines that physical disturbances travel. As a result, a close relationship is maintained between the numerical procedure and the physical phenomena in which the stability of the numerical scheme is easily controlled. The solution is thus, in general, more accurate than other direct finite-difference methods.

The method of characteristics, as applied to problems in two independent variables (referred to as two-variable method) in particular, possesses 
an attractive feature in that the relations holding along characteristics are ordinary differential equations that can easily be handled numerically. When more than two independent variables are involved, however, the simplification is not as great, since the compatibility conditions a re still partial differential equations. The bicharacteristic formulation ${ }^{1-3}$ considers bicharacteristics selected such that the compatibility conditions, when put in a difference form, are essentially like ordinary differential equations. Hence the solution can be constructed without referring directly to conditions outside the physical domain of dependence, i.e., the characteristic cone. On the other hand, the formulation procedure itself is extremely difficult, and, basically, the formulation bears no resemblance to the conventional two-variable method. This is especially true for four or more independent variables.

Saue $r^{4}$ proposed a technique that, basically, represents a direct extension of the two-variable method. It considers lines (Sauer called the se nearcharacteristics) that lie in any plane parallel to the time coordinate. The formulation procedure is similar to the conventional two-variable method, and a problem involving four or more variables presents no more difficulties than the three-variable method. Since the nearcharacteristics, geometrically, are outside the characteristic cone, the solution is directly influenced by conditions outside the true domain of dependence, and it is not clear how this affects the numerical results. A technique proposed by Coburn and Dolph, ${ }^{5}$ further developed by Holt, ${ }^{6}$ involves two bicharacteristics and a line that falls outside the cone. Werner ${ }^{7}$ used the nearcharacteristic formulation in combination with extrapolation methods, and observed that the error in the solution increases as the nearcharacteristic deviates from the bicharacteristic.

This report considers the three-variable formulation that uses the nearcharacteristics lying in spatial coordinate planes. Numerical calculations are carried out for the problem of fluid-hammer wave propagation in a simple geometry, and the results are compared with the results obtained by a bicharacteristic formulation. Comparison is also made with existing analytical solutions and experiments for a plane discontinuity diffracting from a $90^{\circ}$ corner. Acoustic solutions for plane waves and one-dimensional numerical solutions are also presented in support of the numerical results of the nearcharacteristic method. VonNeumann stability tests are performed for the three numerical schemes considered.

\section{BASIC DIFFERENTIAL EQUATIONS}

The basic equations governing the unsteady, compressible, inviscid flows are the conservation equations of mass, momentum, and energy. When the compressibility is small, such as in liquids, the energy equation may be replaced by an isentropic condition with a constant wave speed. The basic differential equations describing the fluid-hammer effects characterizing the 
rapid transients in slightly compressible fluids are written for twodimensional plane and axisymmetric geometry.

Continuity:

$\frac{\partial \rho}{\partial t}+\frac{\partial}{\partial \mathbf{r}}(\rho u)+\nu \frac{\rho u}{r}+\frac{\partial}{\partial z}(\rho w)=0$

Momentum:

$$
\begin{aligned}
& \frac{\partial u}{\partial t}+u \frac{\partial u}{\partial r}+w \frac{\partial u}{\partial z}+\frac{1}{\rho} \frac{\partial p}{\partial r}=0 \\
& \frac{\partial w}{\partial t}+u \frac{\partial w}{\partial r}+w \frac{\partial w}{\partial z}+\frac{1}{\rho} \frac{\partial p}{\partial z}=0 .
\end{aligned}
$$

Isentropic relation:

$$
\left(\frac{\partial p}{\partial p}\right)_{s}=c^{2}
$$

In the se equations, $\nu=0$ for Cartesian and $\nu=1$ for cylindrical geometry. Because of the small change in density, the derivatives of density are eliminated by combining Eqs. 1 and 4:

$$
\frac{\partial p}{\partial t}+u \frac{\partial p}{\partial r}+w \frac{\partial p}{\partial z}+\rho c^{2}\left(\frac{\partial u}{\partial r}+\nu \frac{u}{r}+\frac{\partial w}{\partial z}\right)=0
$$

Equations 2, 3, and 5 are used instead of the original Eqs. 1-4. These equations are first nondimensionalized by using appropriate reference quantities for each variable so that each term is of order of magnitude unity. Although the selection of reference quantities is not always an easy task, a proper nondimensionalization provides a group of dimensionless parameters, physically meaningful, that govern the problem. The nondimensionalized equations are

$$
\begin{aligned}
& \frac{\partial u}{\partial t}+\varepsilon\left(u \frac{\partial u}{\partial r}+\frac{w}{b} \frac{\partial u}{\partial z}\right)+\frac{\partial p}{\partial r}=0, \\
& \frac{\partial w}{\partial t}+\varepsilon\left(u \frac{\partial w}{\partial r}+\frac{w}{b} \frac{\partial w}{\partial z}\right)+\frac{1}{b} \frac{\partial p}{\partial z}=0,
\end{aligned}
$$

and

$$
\frac{\partial p}{\partial t}+e\left(u \frac{\partial p}{\partial r}+\frac{w}{b} \frac{\partial p}{\partial z}\right)+\frac{\partial u}{\partial r}+\nu \frac{u}{r}+\frac{1}{b} \frac{\partial w}{\partial z}=0
$$


where the dimensionless parameters are

$$
\varepsilon=\frac{U}{c}
$$

and

$$
\mathrm{b}=\frac{\mathrm{L}}{\mathrm{R}}
$$

The nondimensionalized variables in Eqs. 6-8 are

$$
\left.\begin{array}{l}
\mathrm{u}=\mathrm{u} * / \mathrm{U} ; \mathrm{w}=\mathrm{w}^{*} / \mathrm{U} ; \mathrm{p}=\mathrm{p}^{*} / \rho \mathrm{cU} ; \\
\mathrm{t}=\mathrm{ct} * / \mathrm{R} ; \mathrm{r}=\mathrm{r}^{*} / \mathrm{R} ; \mathrm{z}=\mathrm{z}^{*} / \mathrm{L} .
\end{array}\right\}
$$

Here, the quantities with an asterisk represent dimensional variables, $c$ is the wave speed, and $R$ and $L$ are the characteristic length in the $r$ and $z$ coordinates, respectively. The reference pressure change $\Delta p$ may be expressed in terms of the reference velocity $U$ as

$$
\Delta \mathrm{p}=\rho \mathrm{cU} .
$$

In the nondimensionalized equations, Eqs. 6-8, the propagation of fluidhammer waves is governed by two dimensionless parameters, $\varepsilon$ is a measure of nonlinear effects, and $\mathrm{b}$ is a geometry parameter. As e approaches a small number, the problem tends to a linear problem. The flow becomes onedimensional for large values of the aspect ratio $b$.

\section{NEARCHARACTERISTIC FORMULATION}

Using the method of nearcharacteristics first proposed by Sauer, ${ }^{4}$ we look for lines that lie in the coordinate planes along which the solution can be extended. (Sauer called these lines nearcharacteristics.) Equations 6-8 are combined linearly to obtain the compatibility conditions that hold along these nearcharacteristics. As derived in Appendix A, two sets each of three compatibility equations are obtained.

In the $\mathrm{r}-\mathrm{t}$ plane:

$$
\begin{aligned}
& d u-d p=\left[\frac{1}{b} \frac{\partial w}{\partial z}+\nu \frac{u}{r}+\frac{\varepsilon w}{b}\left(-\frac{\partial u}{\partial z}+\frac{\partial p}{\partial z}\right)\right] d t \\
& d r=(\varepsilon u-1) d t ; \\
& d z=0
\end{aligned}
$$


$d u+d p=-\left[\frac{1}{b} \frac{\partial w}{\partial z}+\nu \frac{u}{r}+\frac{\epsilon w}{b}\left(\frac{\partial u}{\partial z}+\frac{\partial p}{\partial z}\right)\right] d t ;$

$d r=(\epsilon u+1) d t ;$

$\mathrm{dz}=0$

$d w=-\frac{1}{b}\left(\frac{\partial p}{\partial z}+\epsilon w \frac{\partial w}{\partial z}\right) d t ;$

$\mathrm{dr}=\epsilon \mathrm{udt}$

$\mathrm{d} z=0$.

In the $z-t$ plane:

$d w-d p=\left[\frac{\partial u}{\partial r}+\nu \frac{u}{r}+\epsilon u\left(-\frac{\partial w}{\partial r}+\frac{\partial p}{\partial r}\right)\right] d t ;$

$\mathrm{dr}=0$

$\mathrm{b} d \mathrm{z}=(\epsilon \mathrm{w}-\mathrm{l}) \mathrm{dt}$;

$d w+d p=-\left[\frac{\partial u}{\partial r}+\nu \frac{u}{r}+\epsilon u\left(\frac{\partial w}{\partial r}+\frac{\partial p}{\partial r}\right)\right] d t ;$

$\mathrm{d} \mathbf{r}=0$;

$b d z=(\varepsilon w+1) d t ;$

$d u=-\left(\frac{\partial p}{\partial r}+\epsilon u \frac{\partial u}{\partial r}\right) d t ;$

$\mathrm{dr}=0$;

$b d z=\epsilon w d t$.

Here the operator $d$ represents the differential along the respective nearcharacteristics. The above formulation represents a simple extension of a one-dimensional problem, and it may be easily extended to the threedimensional case.

Figure 1 depicts the projection of nearcharacteristics and the characteristic cone on the current time plane. Also shown is a bicharacteristic, which is a line joining a point on the base circle (with center at $Q$ and radius $\Delta t$ ) and the cone vertex $P$. The four nearcharacteristics- $\overline{1 P}, \overline{2 P}, \overline{3 P}$, and $\overline{4 P}--$ approach bicharacteristics; the remaining two nearcharacteristics approach 
the streamline as the parameter $\varepsilon$ tends to zero. Hence, in the linear limit, the two sets of compatibility relations of the nearcharacteristic method reduce to those of the bicharacteristic formulation, except for the form of the relation along the particle path.

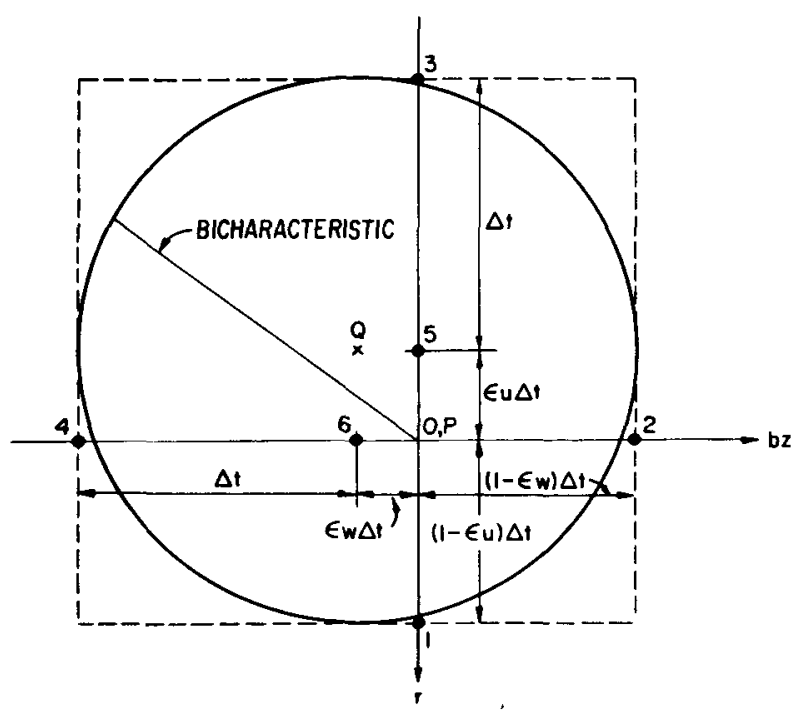

Fig. 1

Projection on $\mathrm{x}-\mathrm{z}$ Plane of Characteristic Cone and Nearcharacteristics (lines connecting $P$ with points $1,2,3,4,5$, and 6). ANL Neg. No. 113-5630.

\section{BOUNDARY AND AXIAL-POINT CONDITION}

On boundaries, some nearcharacteristics fall outside the computational domain. The corresponding compatibility conditions on these nearcharacteristics become unavailable and are replaced by appropriate boundary conditions. The boundary conditions used here are consistent with the inviscid flows. The velocity component normal to rigid boundaries is set to zero, whereas the tangential component is left free. On the axis of symmetry (for $\nu=1)$, i.e., at the axial point, the radial velocity component is zero and the term $u / r$ is replaced by $\partial u / \partial r$.

\section{NUMERICAL PROCEDURE}

For numerical treatment, the differential equations, Eqs. 12-17, are written as finite-difference relations. Since the right-hand side of the compatibility equations contains partial derivatives of all dependent variables, a noniterative second-order scheme cannot be derived. Three first-order schemes are considered here in which the solution is obtained within an error of the order of magnitude of the time step. The first scheme uses equations in the $r-t$ plane, the second scheme uses equations in the $z-t$ plane, and the third scheme uses the leveling technique, in which the above two solutions are averaged with equal weights.

The difference relations in the $r-t$ plane are

$$
u-p=u_{1}-p_{1}+\left[\frac{1}{b} \frac{\partial w}{\partial z}+v \frac{u}{r}+\frac{\epsilon w}{b}\left(-\frac{\partial u}{\partial z}+\frac{\partial p}{\partial z}\right)\right]_{1} \Delta t
$$




$$
\begin{aligned}
& r-r_{1}=\left(\epsilon u_{1}-1\right) \Delta t \\
& u+p=u_{3}+p_{3}-\left[\frac{1}{b} \frac{\partial w}{\partial z}+\nu \frac{u}{r}+\frac{\epsilon w}{b}\left(\frac{\partial u}{\partial z}+\frac{\partial p}{\partial z}\right)\right]_{3} \Delta t \\
& r-r_{3}=\left(\epsilon u_{3}+1\right) \Delta t \\
& w=w_{5}-\frac{1}{b}\left(\frac{\partial p}{\partial z}+\epsilon w \frac{\partial w}{\partial z}\right)_{5} \Delta t \\
& r-r_{5}=\epsilon u_{5} \Delta t .
\end{aligned}
$$

The difference relations in the $z-t$ plane are

$$
\begin{aligned}
& w-p=w_{2}-p_{2}+\left[\frac{\partial u}{\partial r}+\nu \frac{u}{r}+\epsilon u\left(-\frac{\partial w}{\partial r}+\frac{\partial p}{\partial r}\right)\right]_{2} \Delta t ; \\
& b\left(z-z_{2}\right)=\left(\epsilon w_{2}-1\right) \Delta t ; \\
& w+p=w_{4}+p_{4}-\left[\frac{\partial u}{\partial r}+\nu \frac{u}{r}+\epsilon u\left(\frac{\partial w}{\partial r}+\frac{\partial p}{\partial r}\right)\right]_{4} \Delta t ; \\
& b\left(z-z_{4}\right)=\left(\epsilon w_{4}+1\right) \Delta t ; \\
& u=u_{6}-\left(\frac{\partial p}{\partial r}+\epsilon u \frac{\partial u}{\partial r}\right)_{6} \Delta t ; \\
& b\left(z-z_{6}\right)=\epsilon w_{6} \Delta t .
\end{aligned}
$$

Here the variables without subscripts represent the values at the cone vertex $P$ (the unknown point), and the subscripts refer to the intersections of respective nearcharacteristics with the current time plane (see Fig. 1). The coordinates $r_{1}, r_{3}, r_{5}, z_{2}, z_{4}$, and $z_{6}$ can be explicitly determined when the nearcharacteristic relations are combined with interpolation formulas. Hence, the schemes discussed above are truly explicit schemes and require no iterative procedure.

\section{NUMERICAL STABILITY}

The Courant-Friedrichs-Lewy (CFL) criterion ${ }^{8}$ provides the necessary condition for convergence and stability for hyperbolic problems. This condition requires the domain of dependence of the approximate-difference system to contain the domain of dependence of the exact differential system. For the finite-difference grid network shown in Fig. 2, this implies that the time step must be chosen such that all the nearcharacteristics fall inside the rectangle $\mathrm{BCED}$. (No disturbance should traverse more than one grid spacing during one time step.) 


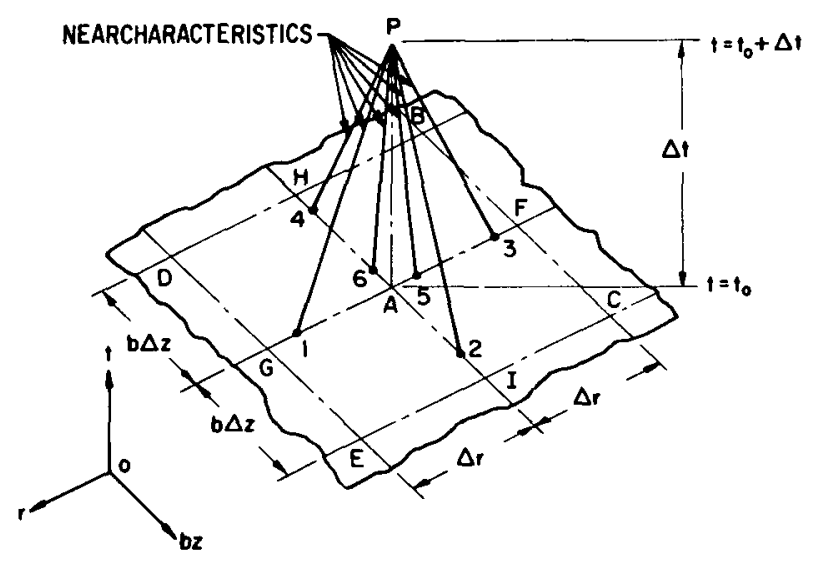

Fig. 2

Finite-difference Network. ANL Neg. No. 113-5627.

The vonNeumann analysis ${ }^{9-11}$ is performed here to find more specific conditions for stability for the three schemes considered. In general, the vonNeumann condition is again a necessary condition. In many instances, however, it is also a sufficient condition. For these solution schemes, explicit expressions are obtained by linear combinations of the compatibility relations. The first scheme (considering the $r-t$ plane) yields, from Eqs. $18 a$, $19 \mathrm{a}$, and $20 \mathrm{a}$,

$$
\begin{aligned}
u= & \frac{1}{2}\left\{u_{1}+u_{3}-p_{1}+p_{3}+\left[\frac{1}{b} \frac{\partial w}{\partial z}+\nu \frac{u}{r}+\frac{\varepsilon w}{b}\left(-\frac{\partial u}{\partial z}+\frac{\partial p}{\partial z}\right)\right]_{1} \Delta t\right. \\
& \left.-\left[\frac{1}{b} \frac{\partial w}{\partial z}+\nu \frac{u}{r}+\frac{\epsilon w}{b}\left(\frac{\partial u}{\partial z}+\frac{\partial p}{\partial z}\right)\right]_{3} \Delta t\right\} ; \\
w= & w_{5}-\frac{1}{b}\left(\frac{\partial p}{\partial z}+\varepsilon w \frac{\partial w}{\partial z}\right)_{5} \Delta t \\
p= & \frac{1}{2}\left\{-u_{1}+u_{3}+p_{1}+p_{3}-\left[\frac{1}{b} \frac{\partial w}{\partial z}+\nu \frac{u}{r}+\frac{\epsilon w}{b}\left(-\frac{\partial u}{\partial z}+\frac{\partial p}{\partial z}\right)\right]_{1} \Delta t\right. \\
& \left.-\left[\frac{1}{b} \frac{\partial w}{\partial z}+\nu \frac{u}{r}+\frac{\epsilon w}{b}\left(\frac{\partial u}{\partial z}+\frac{\partial p}{\partial z}\right)\right]_{3} \Delta t\right\} .
\end{aligned}
$$

The second scheme (considering the $z-t$ plane) is obtained from Eqs. $21 a, 22 a$, and $23 \mathrm{a}$ :

$$
\begin{aligned}
u= & u_{6}-\left(\frac{\partial p}{\partial r}+\epsilon u \frac{\partial u}{\partial r}\right)_{6} \Delta t ; \\
w= & \frac{1}{2}\left\{w_{2}+w_{4}-p_{2}+p_{4}+\left[\frac{\partial u}{\partial r}+\nu \frac{u}{r}+\varepsilon u\left(-\frac{\partial w}{\partial r}+\frac{\partial p}{\partial r}\right)\right]_{2} \Delta t\right. \\
& \left.-\left[\frac{\partial u}{\partial r}+\nu \frac{u}{r}+\epsilon u\left(\frac{\partial w}{\partial r}+\frac{\partial p}{\partial r}\right)\right]_{4} \Delta t\right\}
\end{aligned}
$$




$$
\begin{aligned}
p= & \frac{1}{2}\left\{-w_{2}+w_{4}+p_{2}+p_{4}-\left[\frac{\partial u}{\partial r}+\nu \frac{u}{r}+\epsilon u\left(-\frac{\partial w}{\partial r}+\frac{\partial p}{\partial r}\right)\right]_{2} \Delta t\right. \\
& \left.-\left[\frac{\partial u}{\partial r}+\nu \frac{u}{r}+\epsilon u\left(\frac{\partial w}{\partial r}+\frac{\partial p}{\partial r}\right)\right]_{4} \Delta t\right\} .
\end{aligned}
$$

The vonNeumann tests revealed that these two schemes are numerically unstable for all time steps for any fixed grid sizes. However, the third scheme that yields the solution by averaging the above two unstable schemes is proven stable. The stability condition for the third scheme is found to be

$$
\frac{\Delta \mathrm{r}_{1}}{\Delta \mathrm{r}}+\frac{\Delta \mathrm{r}_{3}}{\Delta \mathrm{r}}+\frac{\Delta \mathrm{z}_{2}}{\Delta \mathrm{z}}+\frac{\Delta \mathrm{z}_{4}}{\Delta \mathrm{z}} \leq 2
$$

where $\Delta r_{1}=\left|r-r_{1}\right|, \Delta r_{3}=\left|r-r_{3}\right|$, etc., and $\Delta r$ and $\Delta z$ are the grid sizes. This condition is equivalent to the stability, requirement determined for the bicharacteristic method. ${ }^{3}$ Numerical calculations, which are discussed in the next section, confirm the findings of the stability analysis. In particular, Eq. 26 is found to be a sufficient condition for the stability of the averaging scheme. Details of the vonNeumann stability analysis are given in Appendix $B$.

\section{NUMERICAL CALCULATIONS}

To test the numerical scheme (scheme 3 that uses the leveling technique) based on the method of nearcharacteristics, an acoustic problem of a

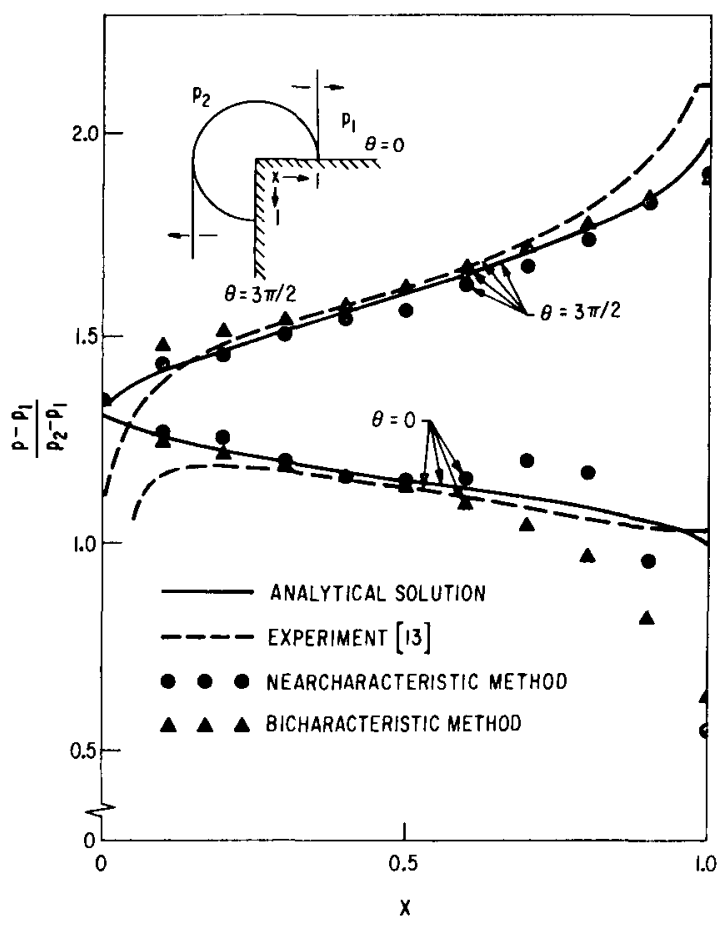

Fig. 3. Diffraction of Plane Acoustic Wave from $90^{\circ}$ Sharp Wedge. ANL Neg. No. 113-5788. plane pressure discontinuity diffracting from a $90^{\circ}$ sharp corner is considered. An analytical solution of this problem was obtained by Keller, ${ }^{12}$ and a comparison was made with the experiment of White and Bleakney. ${ }^{13}$ In Fig. 3, the numerical solution of the nearcharacteristic method is plotted along with the analytical solution and the experiment. Also given in Fig. 3 is the numerical solution obtained by a bicharacteristic method. ${ }^{3}$ The comparison indicates a generally good agreement, except that, near the wave front, some discrepancy appears between the numerical results and the analytical solution due to the numerical dispersion resulting from the restrictive time steps required in both numerical methods for stability and convergence. The discrepancy observed between the experiment and both the analytical and numerical results near the corner is attributed to the viscous vortex effects. The technique 
used in Keller's analytical solution is Busemann's conical flow method ${ }^{14}$ by which the wave equation is transformed into the Laplace's equation. The

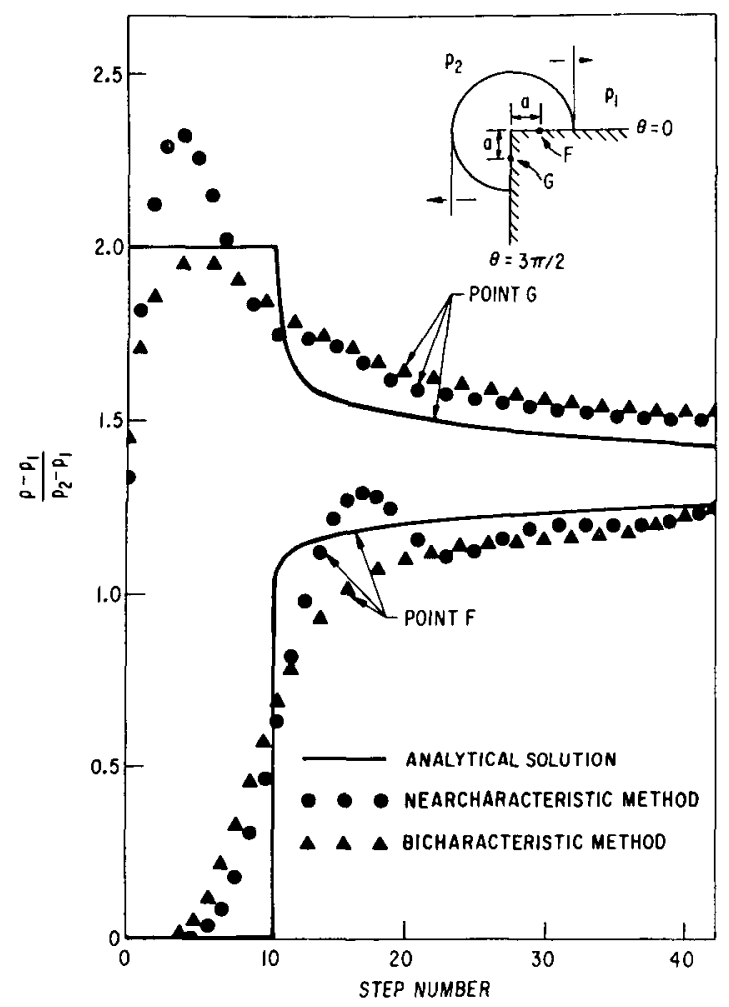

Fig. 4. Pressure-Time History of Points Fixed on Wedge Faces $(\mathrm{c} \Delta \mathrm{t} / \mathrm{a}=0.095 ; \mathrm{c} \Delta \mathrm{t} / \Delta \mathrm{r}=$ $0.475 ; \Delta r=\Delta z)$. ANL Neg. No. 113-5789. potential problem in the circular sector is then solved by a conformal mapping to a unit circle. The experiment of White and Bleakney used a $12 \%$ excess pressure.

A close examination of the nearcharacteristic result in Fig. 3 reveals an oscillatory nature when compared to the other solutions. To examine this more closely, pressure-time histories are given in Fig. 4, in which the pressure at points G and $F$ (located on the wedge faces, distance "a" apart from the corner as shown in Fig. 4) are plotted. Also shown in Fig. 4 for comparison are Keller's analytical solution and the numerical solution based on the bicharacteristic method. The nearcharacteristic result exhibits "overshoots" as well as oscillations about the exact analytical solution, whereas the bicharacteristic result appears to follow the analytical solution without oscillations.

Numerical calculations are further carried out for a problem with a simple geometry involving a sudden expansion and contraction with an axial symmetry.

Figure 5 depicts the geometric configuration. Two different cases with varying aspect ratios ( 0.572 and 2$)$ are considered in examining the varying twodimensionality. Step-pressure pulses are imposed far upstream of the system, which is initially at zero pressure and assumed to be filled with stationary water. Also, the parameter $\epsilon$ is varied $(0,0.0033$, and 0.1$)$ to study the effect of nonlinearity on the numerical results $(\epsilon=0.0033$ and 0.1 correspond to pressure pulses of 1000 and 30,000 psi, respectively).

Fig. 5

Sample Problem. ANL Neg. No. 113-5580.

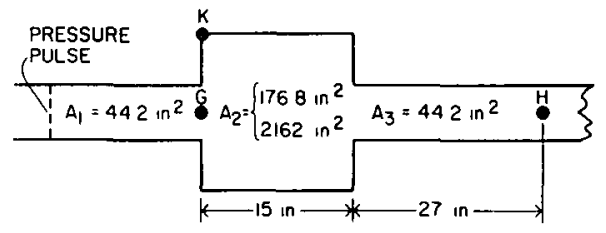

For computational efficiency, the domain is divided into two separate re gions. The two-dimensional region comprises the central part including expansion and contraction, and the rest of the system is treated as one-dimensional. The nearcharacteristic formulation, in the one-dimensional domain, reduces to the more familiar two-variable method (one space coordinate and time). 
At the interfaces that divide the two regions, the simplified conditions $u=$ $\partial \mathrm{u} / \partial \mathrm{r}=\partial \mathrm{w} / \partial \mathrm{r}=\partial \mathrm{p} / \partial \mathrm{r}=0$ are considered. Square grids $(\Delta \mathrm{r}=\mathrm{b} \Delta \mathbf{z})$ are employed (with nine radial nodes for the central region for $b=2$ ) using the time step chosen to meet the vonNeumann condition (Eq. 26).

Figure 6 shows the pressure results at point G (of Fig. 5), in which the three numerical schemes, all using the same time step as determined by Eq. 26, are compared. This and additional calculations, using smaller time steps, revealed that schemes 1 and 2 were unstable for all time steps, while Eq. 26 was the sufficient condition for stability of scheme 3.

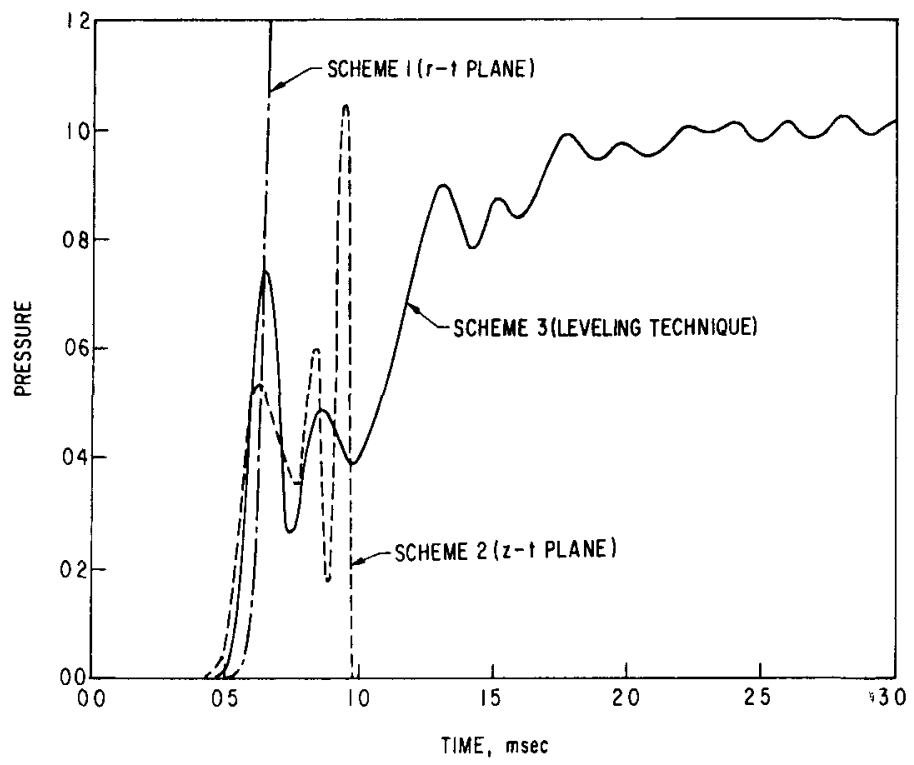

Fig. 6

Nondimensional Pressure at Point $G$ Obtained by Three Numerical Schemes $(\varepsilon=0.0033 ; b=2)$. ANL Neg. No. 113-5631.

Figures 7-9 compare the results of scheme 3 with the results obtained by a bicharacteristic formulation. ${ }^{3}$ The two solutions differ most when large

Fig. 7

Nondimensional Pressure at Point $G$ Comparing Nearcharacteristic with Bicharacteristic Solution $(\varepsilon=0.0033)$. ANL Neg. No. 113-5628.

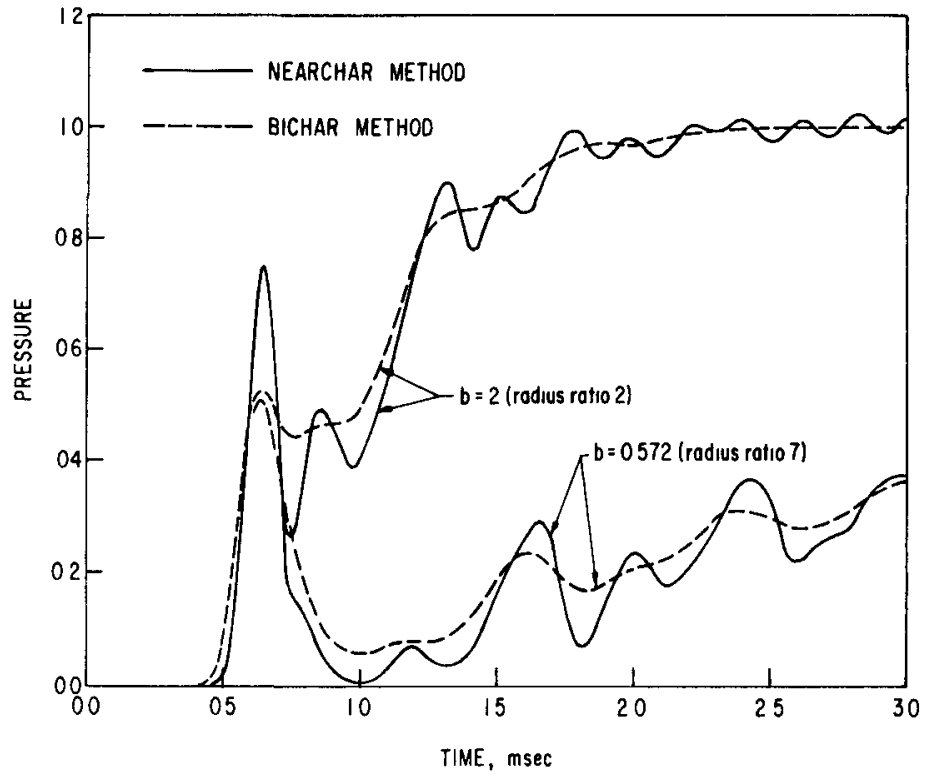




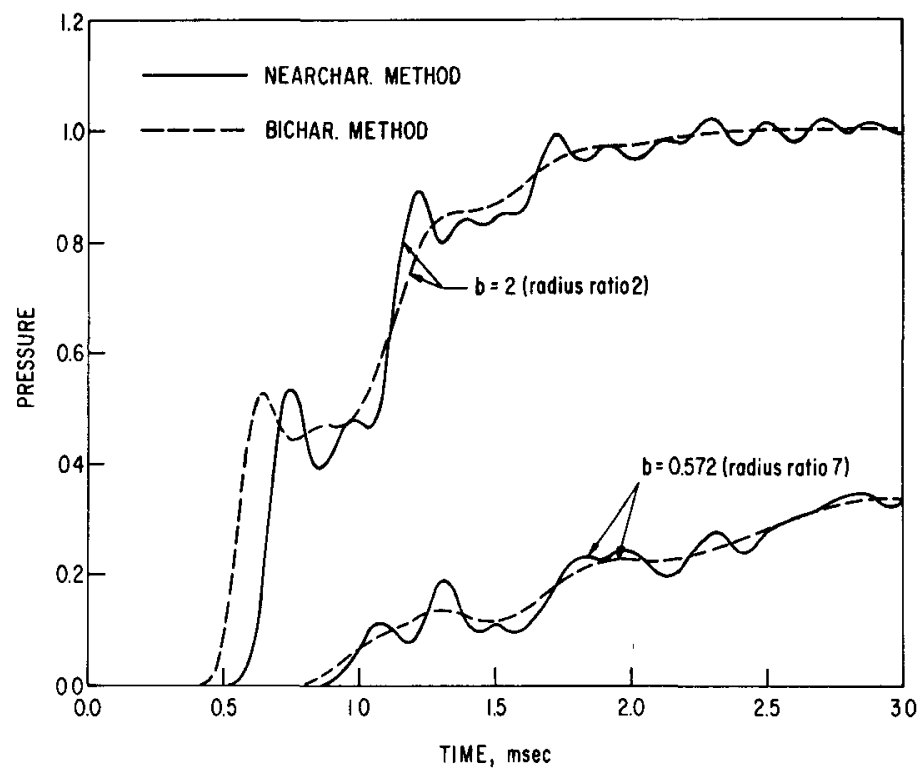

Fig. 8

Nondimensional Pressure at Point $\mathrm{K}$ Comparing Nearcharacteristic with Bicharacteristic Solution $(\varepsilon=0.0033)$. ANL Neg. No. 113-5629.

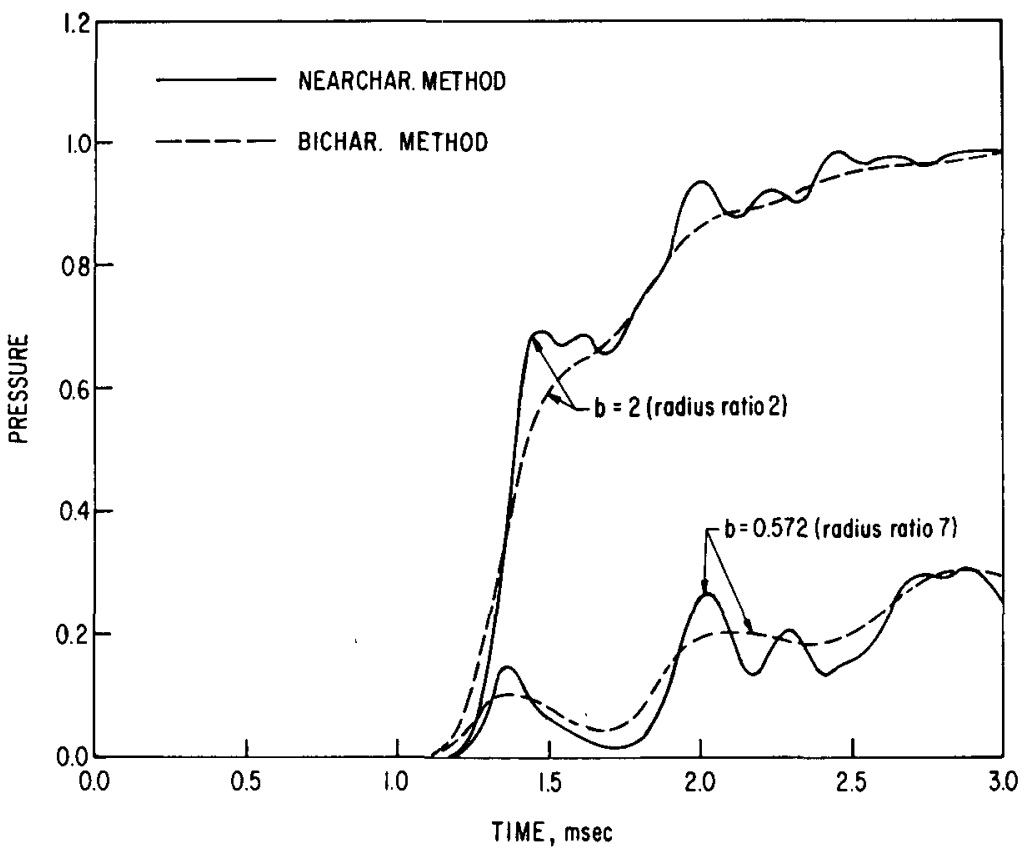

Fig. 9. Nondimensional Pressure at Point $\mathrm{H}$ Comparing Nearcharacteristic with Bicharacteristic Solution $(\varepsilon=0.0033)$. ANL Neg. No. 113-5632.

gradients exist in the solution. As can be seen in Fig. 7, the largest difference occurs at point $G$ during passage of the wave front. Otherwise, the two solutions are in fair agreement. (See, in particular, points $K$ and $H$ in Figs. 8 and 9.) The nearcharacteristic solutions, in general, exhibited an oscillation about the bicharacteristic solution (the same trend as shown in Figs. 3 and 4).

The effect of nonlinearity is shown in Fig. 10, which compares the nonlinear results to the solution of linearized equations (Eqs. 6-8 with $\epsilon=0$ ). 
The result for $\epsilon=0.0033(\Delta p=1000 \mathrm{psi})$ was essentially identical to that for the linear solution and hence not plotted. As the nonlinearity parameter increases to 0.1 , a noticeable difference (up to $18 \%$ ) occurs at points of large gradients. The nonlinear result predicts pressures that exceed the upperlimiting pressure of the linear analysis, i.e., the imposed pressure (nondimensional pressure of 1.0).

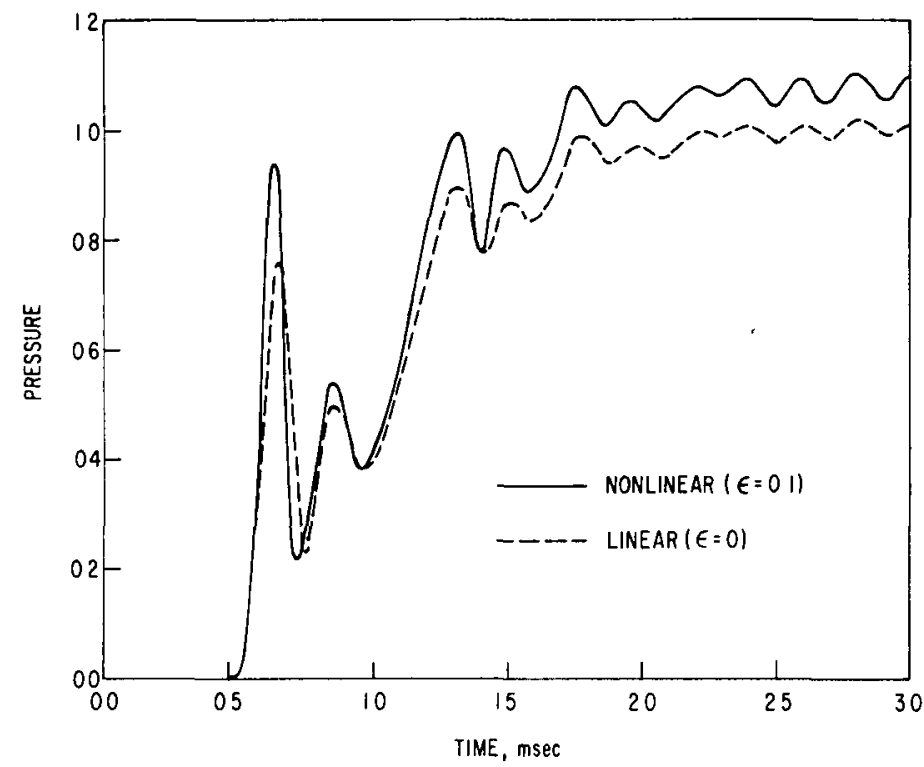

Fig. 10

Nondimensional Pressure at Point $G$ Comparing Nonlinear with Linear Solution $(b=2)$. ANL Neg.

No. $113-5635$.

Figures 11 and 12 compare the two-dimensional nearcharacteristic solutions with the one-dimensional acoustic solution ${ }^{15}$ and the numerical solution based on a one-dimensional treatment for the entire region. The twodimensional result, in general, follows the one-dimensional result for both aspect ratios considered. However, a large discrepancy appears in the expansion region, i.e., at point $G$ for $b=0.572$ (radius ratio 7 ), as the twodimensionality increases (see Fig. ll). At points away from the central region, e.g., at point $\mathrm{H}$, the two-dimensionality is not as large (see Fig. 12).

Fig. 11

Nearcharacteristic Solution at Points $G$ and $K$ in Comparison with One-dimensional Acoustic and Numerical Solutions. ANL Neg. No. 113-5634.

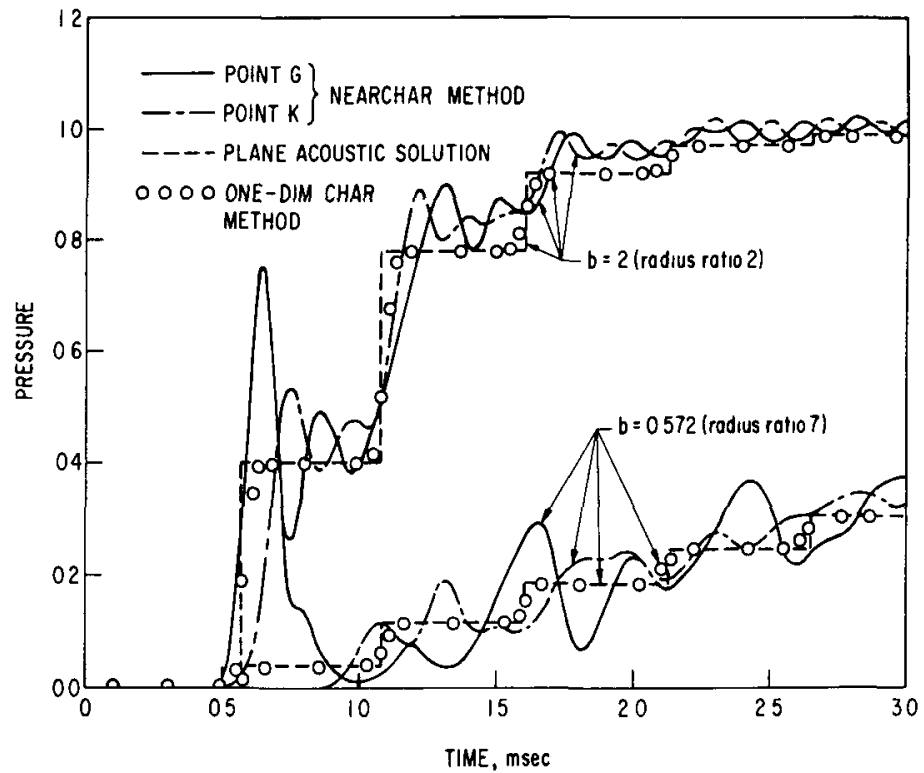




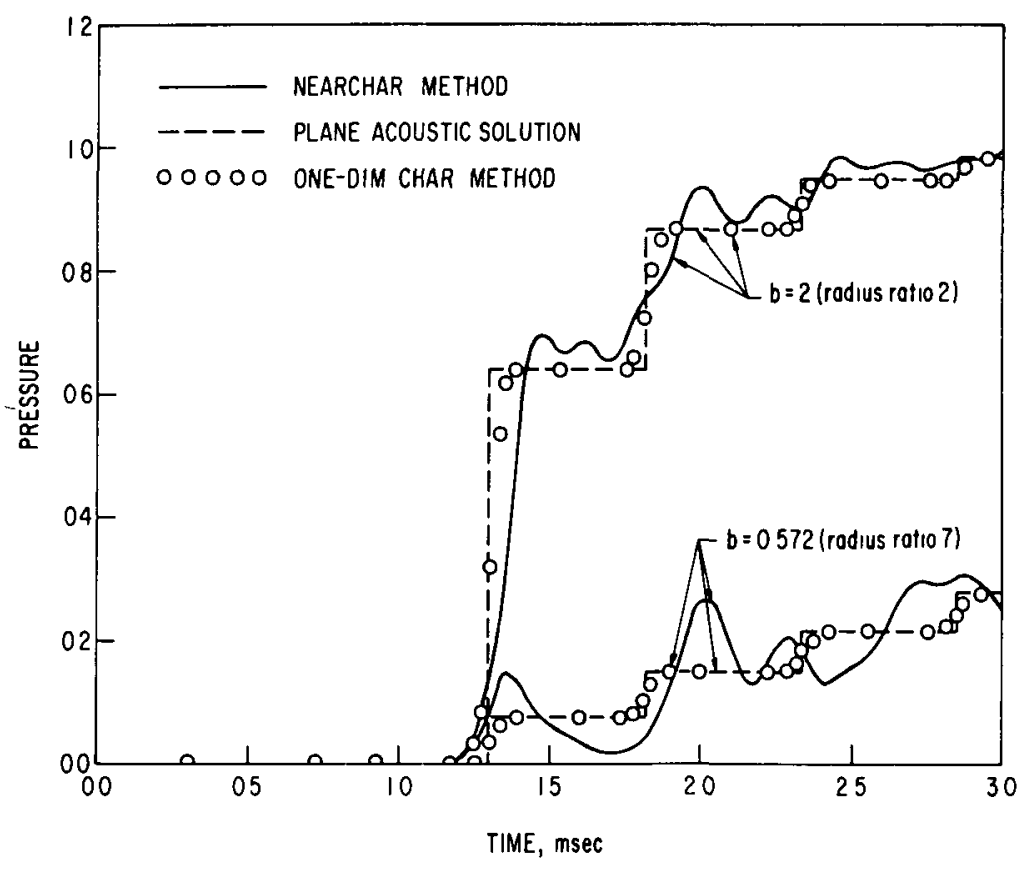

Fig. 12. Nearcharacteristic Solution at Point $\mathrm{H}$ in Comparison with One-dimensional Acoustic and Numerical Solutions. ANL Neg. No. 113-5633.

\section{DISCUSSIONS AND CONCLUSION}

This report discusses the numerical aspects of the nearcharacteristic integration technique through the application of the method to two-dimensional fluid-hammer problems. The nearcharacteristics, along which the numerical solutions are integrated, fell slightly outside the characteristic cone; hence, a direct reference was made to the conditions that have no physical influence on the solution. This is in contrast to the bicharacteristic method, in which only indirect reference is made to regions outside the true domain of dependence due to interpolation requirements. Nevertheless, the nearcharacteristics are admissible directions along which an efficient numerical technique can be formulated. Three numerical schemes were considered. The vonNeumann stability test revealed that schemes that consider nearcharacteristics in any one spatial-coordinate plane, either the $r-t$ or $z-t$ plane, are always unstable, while the scheme that constructs the solution by averaging the unstable schemes is stable. Using the leveling technique, the latter scheme requires the same stability restrictions as the bicharacteristic formulation. The time-step limitation is more stringent than the one dictated by the CFL (Courant-Friedricks-Lewy) criterion.

The numerical results obtained by the nearcharacteristic method exhibited an oscillatory solution about the analytical solution and the bicharacteristic result, the amplitude of oscillation increasing with steepness in the gradient of the solution. The oscillation resembles those that appear in the 
numerical results of direct finite-difference schemes, which are often suppressed by use of artificial viscosity. The method, nevertheless, proved its efficiency, and the accuracy, in general, is demonstrated by comparison with analytical solutions and experiments and also with one-dimensional acoustic solutions in which essentially one-dimensional behavior is expected. The basic technique of treating the entire domain by two separate regions is especially suited to problems involving complex networks in which only limited regions require two-dimensional treatment. For such problems, the technique provides needed resolution in areas of primary interest and, by a smooth transition to the conventional two-variable method, enables the remaining region to be treated one-dimensionally with great reduction in computation time. 


\section{APPENDIX A}

\section{Compatibility Conditions along Nearcharacteristics}

Following the procedures given in Refs. 4 and 16, Eqs. 6-8 are linearly combined:

$$
\begin{gathered}
\alpha_{1}\left[\frac{\partial u}{\partial t}+\epsilon\left(u \frac{\partial u}{\partial r}+\frac{w}{b} \frac{\partial u}{\partial z}\right)+\frac{\partial p}{\partial r}\right]+\alpha_{2}\left[\frac{\partial w}{\partial t}+\epsilon\left(u \frac{\partial w}{\partial r}+\frac{w}{b} \frac{\partial w}{\partial z}\right)+\frac{1}{b} \frac{\partial p}{\partial z}\right] \\
+\alpha_{3}\left[\frac{\partial p}{\partial t}+\epsilon\left(u \frac{\partial p}{\partial r}+\frac{w}{b} \frac{\partial p}{\partial z}\right)+\frac{\partial u}{\partial r}+v \frac{u}{r}+\frac{1}{b} \frac{\partial w}{\partial z}\right]=0
\end{gathered}
$$

Consider first the $r-t$ plane. Equation A. 1 is rearranged so that the left-hand side is in a characteristic form, with the characteristics lying in the $r-t$ plane:

$$
\begin{aligned}
\alpha_{1} \frac{\partial \mathrm{u}}{\partial \mathrm{t}}+\left(\alpha_{1} \varepsilon \mathrm{u}+\alpha_{3}\right) \frac{\partial \mathrm{u}}{\partial \mathrm{r}}+\alpha_{2} \frac{\partial \mathrm{w}}{\partial \mathrm{t}}+\alpha_{2} \varepsilon \mathrm{u} \frac{\partial \mathrm{w}}{\partial \mathrm{r}}+\alpha_{3} \frac{\partial \mathrm{p}}{\partial \mathrm{t}}+\left(\alpha_{3} \varepsilon \mathrm{u}+\alpha_{1}\right) \frac{\partial \mathrm{p}}{\partial \mathrm{r}}=-\alpha_{1} \epsilon \frac{\mathrm{w}}{\mathrm{b}} \frac{\partial \mathrm{u}}{\partial \mathrm{z}} \\
-\alpha_{2} \frac{1}{\mathrm{~b}}\left(\epsilon \mathrm{w} \frac{\partial \mathrm{w}}{\partial \mathrm{z}}+\frac{\partial \mathrm{p}}{\partial \mathrm{z}}\right)-\alpha_{3}\left[\nu \frac{\mathrm{u}}{\mathrm{r}}+\frac{1}{\mathrm{~b}}\left(\frac{\partial \mathrm{w}}{\partial \mathrm{z}}+\epsilon \mathrm{w} \frac{\partial \mathrm{p}}{\partial \mathrm{z}}\right)\right] .
\end{aligned}
$$

It follows that

$$
\frac{\mathrm{dr}}{\mathrm{dt}}=\frac{1}{\alpha_{1}}\left(\alpha_{1} \in \mathrm{u}+\alpha_{3}\right)=\frac{1}{\alpha_{2}} \alpha_{2} \varepsilon \mathrm{u}=\frac{1}{\alpha_{3}}\left(\alpha_{3} \in \mathrm{u}+\alpha_{1}\right)
$$

where the operator $d$ denotes the differential along a nearcharacteristic in the $\mathbf{r}-t$ plane. Equation A.3 yields three equations that are homogeneous in the $\alpha^{\prime} s$. The existence of real characteristics requires the coefficient determinant (called the characteristic determinant) to vanish:

$$
\left|\begin{array}{ccc}
\varepsilon u-\frac{d r}{d t} & 0 & 1 \\
0 & \varepsilon u-\frac{d r}{d t} & 0 \\
l & 0 & \varepsilon u-\frac{d r}{d t}
\end{array}\right|=0 .
$$

This yields three nearcharacteristic equations:

$$
\frac{\mathrm{dr}}{\mathrm{dt}}=\epsilon \mathrm{u}-1
$$




$$
\frac{\mathrm{dr}}{\mathrm{dt}}=\varepsilon u+1
$$

and

$$
\frac{\mathrm{dr}}{\mathrm{dt}}=\epsilon \mathrm{u}
$$

Substitution of these equations into Eq. A. 3 determines three sets of values for the $\alpha^{\prime} s$. The corresponding compatibility equations are then determined from Eq. A. 1:

$$
\begin{aligned}
& \frac{\partial u}{\partial t}+(\epsilon u-1) \frac{\partial u}{\partial r}-\frac{\partial p}{\partial t}-(\epsilon u-1) \frac{\partial p}{\partial r}=\frac{1}{b} \frac{\partial w}{\partial z}+v \frac{u}{r}+\frac{\epsilon w}{b}\left(-\frac{\partial u}{\partial z}+\frac{\partial p}{\partial z}\right) \\
& \frac{\partial u}{\partial t}+(\epsilon u+1) \frac{\partial u}{\partial r}+\frac{\partial p}{\partial t}+(\epsilon u+1) \frac{\partial p}{\partial r}=-\frac{1}{b} \frac{\partial w}{\partial z}-v \frac{u}{r}-\frac{\epsilon w}{b}\left(\frac{\partial u}{\partial z}+\frac{\partial p}{\partial z}\right)
\end{aligned}
$$

and

$$
\frac{\partial w}{\partial t}+\epsilon u \frac{\partial w}{\partial r}=-\frac{1}{b} \frac{\partial p}{\partial z}-\frac{\epsilon w}{b} \frac{\partial w}{\partial z}
$$

A similar procedure determines the following three compatibility equations in the $\mathrm{z}-\mathrm{t}$ plane:

$$
\begin{aligned}
& \frac{\partial w}{\partial t}+\frac{1}{b}(\epsilon w-1) \frac{\partial w}{\partial z}-\frac{\partial p}{\partial t}-\frac{1}{b}(\epsilon w-1) \frac{\partial p}{\partial z}=\frac{\partial u}{\partial r}+\nu \frac{u}{r}+\epsilon u\left(-\frac{\partial w}{\partial r}+\frac{\partial p}{\partial r}\right) \\
& \frac{\partial w}{\partial t}+\frac{1}{b}(\epsilon w+1) \frac{\partial w}{\partial z}+\frac{\partial p}{\partial t}+\frac{1}{b}(\epsilon w+1) \frac{\partial p}{\partial z}=-\frac{\partial u}{\partial r}-\nu \frac{u}{r}-\epsilon u\left(\frac{\partial w}{\partial r}+\frac{\partial p}{\partial r}\right)
\end{aligned}
$$

and

$$
\frac{\partial u}{\partial t}+\frac{\epsilon w}{b} \frac{\partial u}{\partial z}=-\frac{\partial p}{\partial r}-\epsilon u \frac{\partial u}{\partial r}
$$




\section{APPENDIX B}

Fourier Stability Analysis of vonNeumann

Since the Fourier analysis can only be performed for linear systems, the numerical schemes described by Eqs. 24 and 25 are first linearized by assuming small convective terms (i.e., $\epsilon \rightarrow 0$ in the equations). This brings forth further simplifications that

$$
\left|r-r_{1}\right|=\left|r-r_{3}\right|=b\left|z-z_{2}\right|=b\left|z-z_{4}\right|=\delta \Delta r
$$

and

$$
\left|r-r_{5}\right|=b\left|z-z_{6}\right|=0
$$

where $\Delta \mathrm{r}=\mathrm{b} \Delta \mathrm{z}$ is also assumed and $\delta$ is defined by Eq. B.la. The procedure outlined in the following is limited to the plane two-dimensional case $(\nu=0)$. The axisymmetric case can be treated essentially in the same manner, and the results obtained for the plane case apply equally to the axisymmetric case.

The dependent variables are written as Fourier series, and each component is examined as to its growth with time. A Fourier component of the variable $u$, for example, at the advanced and current time level and at location $\mathbf{r}=\mathrm{j} \Delta \mathbf{r}, \mathbf{z}=\mathrm{k} \Delta \mathrm{z}$, can be written as

$$
u(j \Delta r, k \Delta z, t+\Delta t)=u_{0}^{n+1} e^{i \beta j \Delta r} e^{i \gamma k \Delta z}
$$

and

$$
u(j \Delta r, k \Delta z, t)=u_{0}^{n} e^{i \beta j \Delta r} e^{i \gamma k \Delta z},
$$

where $\beta$ and $Y$ are the wave numbers associated with the $r$ and $z$ coordinates, and $u_{0}^{n+1}$ and $u_{0}^{n}$ are the time factors at the advanced and current time level, respectively. Similar expressions are considered for the other two variables. The substitution of these expressions into the linearized version of the numerical schemes and simplification yields linear systems of the time factors in the form

$$
\left(\begin{array}{c}
u_{0}^{n+1} \\
w_{0}^{n+1} \\
p_{0}^{n+1}
\end{array}\right)=A(\beta \Delta r, y \Delta z, \Delta t)\left(\begin{array}{c}
u_{0}^{n} \\
w_{0}^{n} \\
p_{0}^{n}
\end{array}\right),
$$

where $A$ is a matrix called the amplification matrix. Following vonNeumann (as given in Refs. 9-11), the numerical stability requires the spectral radius of the amplification matrix (i.e., the maximum of the absolute value of the eigenvalues) not to exceed unity. 
In deriving the finite-difference form of the solution schemes, Eqs. 24 and 25 , the values of the dependent variables $\left(u_{1}, u_{2}, \ldots\right.$, etc. $)$ and their derivatives $(\partial u / \partial r, \partial w / \partial r, \ldots$, etc. $)$ in the current time plane are evaluated by linear interpolations. For example, $u_{1}=\delta u_{G}+(1-\delta) u_{A}, \ldots,(\partial u / \partial r)_{2}=\delta\left(u_{E}-u_{C}\right) /$ $2 \Delta \mathbf{r}+(1-\delta)\left(u_{G}-u_{F}\right) / 2 \Delta r, \ldots$, etc., where the subscripts refer to specific grid points in the current time plane as shown in Fig. 2. The amplification matrix $A_{1}$ of the linearized version of the first scheme is thus obtained:

$$
A_{1}=\left(\begin{array}{ccc}
1 & 0 & -i \delta \sin \beta \Delta r \\
-\delta^{2}(\sin \beta \Delta r)(\sin \gamma \Delta z) & 1-\delta(1-\cos \gamma \Delta z) & -i \delta \sin \gamma \Delta z \\
-i \delta(\sin \beta \Delta r) & -i \delta \sin \gamma \Delta z & 1-\delta(1-\cos \gamma \Delta z) \\
{[1-\delta(1-\cos \gamma \Delta z)]} & - &
\end{array}\right) .
$$

The eigenvalues $\lambda$ of this matrix are determined by the following cubic equation:

$$
\begin{aligned}
& (1-\lambda)\left[(1-\delta+\delta \cos \gamma \Delta z-\lambda)^{2}+\delta^{2} \sin ^{2} Y \Delta z\right]+\delta^{2}\left(\sin ^{2} \beta \Delta r\right)\left[\delta^{2} \sin ^{2} \gamma \Delta z\right. \\
& +(1-\delta+\delta \cos Y \Delta z)(1-\delta+\delta \cos \gamma \Delta z-\lambda)]=0 .
\end{aligned}
$$

It is difficult to solve Eq. B. 5 for all possible values of $\beta \Delta r$ and $\gamma \Delta z$. Nevertheless, it is possible to show that, for some particular choice of these values, the eigenvalue takes on a number that is greater than unity. Indeed, for the choice of $\sin \gamma \Delta z=0$ and $\cos \gamma \Delta z=1$,

$$
\lambda=1 \pm \mathrm{i} \delta \sin \beta \Delta \mathrm{r} \text {. }
$$

Hence,

$$
|\lambda|=\sqrt{1+\delta^{2} \sin ^{2} \beta \Delta r},
$$

which clearly indicates that the eigenvalue exceeds unity for all values of $\delta>0$. This is a sufficient proof that scheme 1 is unconditionally unstable.

From the complete symmetry of the two schemes, Eqs. 24 and 25, the result for the linearized system of scheme 2 can immediately be written down:

$$
|\lambda|=\sqrt{1+\delta^{2} \sin ^{2} \gamma \Delta z}
$$

which again proves the instability of scheme 2 .

A procedure similar to the above is applied to scheme 3 (that uses the averaging technique), from which the following results are obtained: the amplification matrix, 


$$
A_{3}=\frac{1}{2}\left(\begin{array}{ccc}
2-\delta(1-\cos \beta \Delta r) & -\delta^{2}(\sin \beta \Delta r)(\sin \gamma \Delta z) & -i 2 \delta \sin \beta \Delta r \\
-\delta^{2}(\sin \beta \Delta r)(\sin \gamma \Delta z) & 2-\delta(1-\cos \gamma \Delta z) & -i 2 \delta \sin \gamma \Delta z \\
-i \delta(\sin \beta \Delta r) & -i \delta(\sin \gamma \Delta z) & 2-\delta(2-\cos \beta \Delta r \\
{[2-\delta(1-\cos \gamma \Delta z)]} & {[2-\delta(1-\cos \beta \Delta r)]} & -\cos \gamma \Delta z)
\end{array}\right) ;
$$

and the third-order polynomial for the eigenvalues,

$$
\begin{aligned}
& {[2(1-\lambda)-\delta(1-\cos \beta \Delta r)]\{[2(1-\lambda)-\delta(1-\cos \gamma \Delta z)][2(1-\lambda)} \\
&\left.-\delta(2-\cos \beta \Delta r-\cos \gamma \Delta z)]+2 \delta^{2} \sin ^{2} \gamma \Delta z[2-\delta(1-\cos \beta \Delta r)]\right\} \\
&+\delta^{4}\left(\sin ^{2} \beta \Delta r\right)\left(\sin ^{2} \gamma \Delta z\right)\{2[2-\delta(1-\cos \delta \Delta z)]+2[2-\delta(1-\cos \beta \Delta r)] \\
&-[2(1-\lambda)-\delta(2-\cos \beta \Delta r-\cos \gamma \Delta z)]\}+2 \delta^{2}\left(\sin ^{2} \beta \Delta r\right)\{[2-\delta(1 \\
&-\cos \gamma \Delta z)][2(1-\lambda)-\delta(1-\cos \gamma \Delta z)]\}=0 .
\end{aligned}
$$

The general solution of this polynomial again is extremely difficult; hence, various specific values of $\beta \Delta r$ and $\gamma \Delta z$ were considered. It was indicated that the most stringent condition on $\delta$ results from the choice $\beta \Delta r=0$ (so that $\sin \beta \Delta r=0$ and $\cos \beta \Delta r=1$ ). Thus,

$$
\lambda=\left[1-\frac{\delta}{2}(1-\cos \gamma \Delta z)\right] \pm i \delta \sin \gamma \Delta z
$$

Finally, the requirement $|\lambda| \leq 1$ yields the condition for stability of the third scheme:

$$
2 \delta \leqslant 1
$$

If the simplifications, Eqs. B.l, were not made, the above procedure would involve a more complicated algebra. Nevertheless, it can be shown that the stability condition of the third scheme that accounts for the nonlinear characteristic equations is indeed the one described by Eq. 26. 


\section{REFERENCES}

1. D. S. Butler, The Numerical Solution of Hyperbolic Systems of Partial Differential Equations in Three Independent Variables, Proc. Roy. Soc. London, Series A 255, 232-252 (1960).

2. D. J. Richardson, "Solutions of Two-Dimensional Hydrodynamic Equations by the Method of Characteristics," in Methods in Computational Physics, Vol. 3, B. Alder, S. Fernbach, and M. Rotenberg, eds., Academic Press, New York, pp. 295-318 (1964).

3. Y. W. Shin and R. A. Valentin, Two-dimensional Fluid-hammer Analysis by the Method of Characteristics in a Closed Axisymmetric Cylindrical Domain, ANL-8090 (Aug 1974); Y. W. Shin and R. A. Valentin, Numerical Analysis of Fluid-hammer Waves by the Method of Characteristics, submitted for publication in J. Comp. Phys.

4. R. Sauer, Differenzenverfahren für hyperbolische Anfangswert-probleme bei mehr als zwei unabhängigen Veränderlichen mit Hilfe von Nebencharakteristiken, Num. Math. 5, 55-67 (1963).

5. N. Coburn and C. L. Dolph, "The Method of Characteristics in the ThreeDimensional Stationary Supersonic Flow of a Compressible Gas," Proc. Ist Symp. Appl. Math., Amer. Math. Soc., pp. 55-66 (1949).

6. M. Holt, The Method of Characteristics for Steady Supersonic Rotational Flow in Three Dimensions, J. Fluid Mech. 1, 409-423 (1956).

7. W. Werner, Numerical Solution of Systems of Quasizinear Hyperbolic Differential Equations by Means of the Method of Nebencharacteristics in Combination with Extrapolation Methods, Num. Math. 11, 151-169 (1968).

8. R. Courant, K. Friedrichs, and H. Lewy, Über die Partielzen Differenzialgleichungen der Mathematischen Physik, Math. Ann. 100, 32-74 (1928).

9. G. G. O'Brien, M. A. Hyman, and S. Kaplan, A Study of the Numerical Solution of Partial Differential Equations, J. Math. Phys. 29, 223-251 (1950).

10. R. D. Richtmyer and K. W. Morton, Difference Methods for Initial-VaZue Problems, 2nd ed., Interscience Publishers, New York (1967).

11. P. Fox, "The Solution of Hyperbolic Partial Differential Equations by Difference Methods," in Mathematical Methods for Digital Computers, A. Ralston and H. S. Wilf, eds., Chap. 16, John Wiley and Sons, Inc., New York/London, pp . 180-188 (1960).

12. J. Keller, Diffraction of a Shock or an Electromagnetic Pulse by a RightAngled Wedge, J. App1. Phys. 23, 1267-1268 (1952).

13. D. R. White and W. Bleakney, Shock Loading of Rectangular Structure, Technical Report II-11, Dept. of Physics, Princeton University (Jan 1952).

14. A. Busemann, Infinitesimal Conical Supersonic Flow, Schriften der Deutschen Akademie für Luftfahrtforschung, Vol. 7B, No. 3, 105-122 (1953).

15. L. E. Kinsler and A. R. Frey, Fundamentals of Acoustics, 2nd ed., John Wiley and Sons, Inc., New York (1962).

16. C. A. Kot and Y. W. Shin, Formulation of the Nearcharacteristic Method for Multidimensional Fluid-hammer Problems, Technical Memorandum, ANL-CT-75-02 (Aug 1974). 